\title{
Predictive value of ADAMTS- 3 on concealed chronic renal failure in COPD patients
}

This article was published in the following Dove Press journal: International Journal of COPD

\section{Mian Zeng \\ Qingui Chen \\ Wenjie Liang \\ Wanmei He \\ Haichong Zheng \\ Chunrong Huang}

Department of Medical Intensive Care Unit, the First Affiliated Hospital of Sun Yat-Sen University, Guangzhou, China
Correspondence: Mian Zeng Department of Medical Intensive Care Unit, the First Affiliated Hospital of Sun Yat-Sen University, No 58 Zhongshan 2nd Road, Yuexiu District, Guangzhou 51 0080, Guangdong Province, China Tel +86208775 5766

Email zengmian2004@I63.com
Background: Impaired renal function is often neglected in COPD patients. Considering that COPD patients usually have an ongoing prothrombotic state and systemic inflammation status, we investigated the association among them and explored the predictive value of a disintegrin and metalloproteinase with a thrombospondin type 1 motif, member 13 (ADAMTS-13), on concealed chronic renal failure (CRF) in COPD patients.

Methods: COPD patients were recruited from the First Affiliated Hospital of Sun Yat-Sen University between January 2015 and December 2016. Control was selected from contemporaneous hospitalized patients without COPD and matched by age and gender at a ratio of 1:1. Estimated glomerular filtration rate (eGFR) was calculated by using the Chronic Kidney Disease Epidemiology Collaboration formula, and all subjects were categorized as having normal renal function (eGFR $\geq 60 \mathrm{~mL} \mathrm{~min}^{-1} 1.73 \mathrm{~m}^{-2}$ ) and having concealed CRF (normal serum creatinine

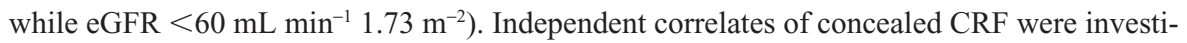
gated by logistic regression analysis, and receiver operating characteristic (ROC) curves were used to determine the predictive value of ADAMTS-13.

Results: In total, 106 COPD and 106 non-COPD patients were finally recruited, and the incidences of concealed CRF were 19.81\% and 7.55\%, respectively. ADAMTS-13 (odds ratio $[\mathrm{OR}]=0.858,95 \% \mathrm{CI}=0.795-0.926)$, $\mathrm{D}$-dimer $(\mathrm{OR}=1.095,95 \% \mathrm{CI}=1.027-1.169)$, and C-reactive protein $(\mathrm{OR}=1.252,95 \% \mathrm{CI}=1.058-1.480)$ were significantly associated with concealed CRF. Sensitivity and specificity at an ADAMTS-13 cutoff of $318.72 \mathrm{ng} / \mathrm{mL}$ were $100 \%$ and $81.2 \%$, respectively. The area under the ROC curve was 0.959 . Conclusion: Prothrombotic state and systemic inflammation status might contribute to explaining the high incidence of concealed CRF in COPD, and plasma ADAMTS-13 levels may serve as a strong predictor.

Keywords: chronic obstructive pulmonary disease, renal insufficiency, thrombosis, inflammation, ADAMTS-13 protein

\section{Background}

COPD, a disease characterized by persistent and progressing airflow limitation, presents not only pulmonary changes, but also various extrapulmonary effects and comorbidities including cardiovascular disease, osteoporosis, depression, and diabetes. ${ }^{1}$ With increased evidence, it is gradually realized that chronic renal failure (CRF) is not a rare but neglected comorbidity in COPD patients. ${ }^{2,3}$ However, currently, studies on CRF in COPD patients are still limited. Therefore, it seems to be difficult to identify COPD patients combined with CRF earlier, especially those with concealed CRF who have normal serum creatinine levels, but decreased estimated glomerular filtration rate (eGFR). Considering that COPD patients are usually accompanied with hypercoagulability, elevated levels of fibrinolytic markers, and systematic 
inflammation status, it remains unknown whether there is an association among prothrombotic state, inflammation status, and concealed CRF. ${ }^{4-7}$ It has been reported that a disintegrin and metalloproteinase with a thrombospondin type 1 motif, member 13 (ADAMTS-13) can modulate the decomposition of von Willebrand factor (vWF), which is closely related to microvascular thrombosis. ${ }^{8}$ Meanwhile, some studies indicated that ADAMTS-13 possessed an inflammatory regulation effect. ${ }^{9}$ Thus, ADAMTS-13 might be valuable in predicting concealed CRF in COPD. Therefore, we analyzed the levels of ADAMTS-13, D-dimer, fibrinogen (Fbg), and some other biomarkers that potentially correlate with prothrombotic state or inflammation status, aiming at investigating the association among prothrombotic state, inflammation status, and concealed CRF and determining the predictive value of ADAMTS-13 for CRF in COPD patients. ${ }^{4,10}$

\section{Methods}

\section{Patients}

Patients diagnosed with COPD according to the Global Initiative for Chronic Obstructive Lung Disease were recruited from the inpatient and outpatient respiratory units of the First Affiliated Hospital of Sun Yat-Sen University between January 2015 and December 2016. ${ }^{1}$ Potential subjects who had any of the following were excluded from the study: definitive history of renal toxic drug use; highly susceptible or determined cancer; history of renal operation or renal injury; coexistence with primary glomerular diseases, acute renal injury, or hereditary renal disease; need for renal replacement therapy; coexistence with asthma, bronchiectasis, pulmonary tuberculosis, or other chronic respiratory diseases; coexistence with other chronic diseases that might cause renal injury, such as diabetes mellitus and hypertension; coexistence with blood disorders; history of receiving antithrombotic therapy in the past 3 months; and a high serum creatinine level. Non-COPD patients against the exclusion criteria above were enrolled from other medical departments in the same period as a control group matched on age $( \pm 2)$ and gender by a ratio of $1: 1$. The study was approved by the Ethics Committee of the First Affiliated Hospital of Sun Yat-Sen University prior to study initiation. All the participants provided informed signed consent. This study did not meet the International Committee of Medical Journal Editors/World Health Organization clinical trial definition; therefore, registration was not required.

\section{Measurements}

Baseline information such as personal information and smoking history was recorded for all included patients.
Body mass index was calculated based on height and weight, and blood levels of creatinine, vWF, mean platelet volume (MPV), platelet distribution width (PDW), Fbg, D-dimer, interleukin-6 (IL-6), and C-reactive protein (CRP) were measured routinely by the clinical laboratory within 24 hours after admission. At the same time, plasma ADAMTS-13 levels were determined by using enzyme-linked immunosorbent assay kits developed by the Sekisui Diagnostics Inc. (Maidstone, UK), and pulmonary function tests were conducted by the pulmonary physiology laboratory. The GFR was estimated by using the Chronic Kidney Disease Epidemiology Collaboration (CKD-EPI) equation based on age, gender, and serum creatinine level, and then, the patients included were divided into two groups: one was the concealed CRF group (eGFR $<60 \mathrm{~mL} \mathrm{~min}^{-1} 1.73 \mathrm{~m}^{-2}$ ), and the other was the normal renal function group (eGFR $\geq 60 \mathrm{~mL} \mathrm{~min}^{-1}$ $\left.1.73 \mathrm{~m}^{-2}\right) .^{11}$

\section{Statistical analysis}

The results are expressed as mean \pm SD or median (interquartile range) for continuous variables and the number of patients with percentages for categorical variables. SPSS Statistics Version 24.0 (IBM Corporation, Armonk, NY, USA) was used for data processing and analysis. Data between groups were compared by using Student's $t$-test, Wilcoxon rank sum test, or $\chi^{2}$ test determined by the type of data. Coexistence with concealed CRF was chosen as the response variable, and a univariate logistic model was used to test all the variables mentioned above. Variables that have shown a $P$-value $\leq 0.05$ were included in a multivariate model afterward to examine the multivariable mixed effect of different explanatory variables on the response variable. The same analysis was conducted in the COPD patients alone. Two-tailed $P$-values $<0.05$ were considered significant. Receiver operating characteristic (ROC) curves were used to determine the predictive value of ADAMTS-13 and further to find the test cutoff value determined by the point where the Youden's index is maximum.

\section{Results \\ Baseline data}

In total, 106 COPD patients and 106 non-COPD patients were included finally with an average age of $69.48 \pm 9.15$ and $69.27 \pm 9.47$, respectively, and the same proportion of men $87.74 \%$ (93 of 106). The incidences of concealed CRF were $19.81 \%$ (21 of 106) and 7.55\% (8 of 106), respectively, among the COPD patients and non-COPD patients included. It showed significant differences in the proportion of smokers, the blood level of ADAMTS-13, vWF, MPV, PDW, Fbg, 
D-dimer, IL-6, CRP, and some parameters of lung function between COPD patients and non-COPD patients. Table 1 shows the details of characteristics of the two groups.

\section{Association among prothrombotic state, inflammation, and concealed CRF}

The results from univariate logistic regression analysis, as shown in Table 2, indicated that elevated levels of vWF, Fbg, D-dimer, and CRP might be the risk factors for concealed CRF, while an elevated level of ADAMTS-13 might be a protective factor. After adjusting for confounding variables, ADAMTS-13, D-dimer, and CRP were closely associated with concealed CRF. D-dimer (odds ratio $[\mathrm{OR}]=1.095,95 \% \mathrm{CI}=1.027-1.169)$ and $\mathrm{CRP}$ ( $\mathrm{OR}=1.252,95 \% \mathrm{CI}=1.058-1.480)$ might be risk factors, while ADAMTS-13 (OR $=0.858,95 \%$ CI $=0.795-0.926)$ might be a protective factor (Table 3 ). Subgroup analysis of COPD subjects alone showed a similar result (Tables 2 and 3 ).

\section{Predictive value of ADAMTS- 13}

Figure 1 presented the ROC curves for ADAMTS-13 in predicting concealed $\mathrm{CRF}$ in COPD patients, and the area

Table I Baseline data of included patients

\begin{tabular}{|c|c|c|c|c|}
\hline & $\begin{array}{l}\text { Non-COPD } \\
\text { patients } \\
(n=106)\end{array}$ & $\begin{array}{l}\text { COPD } \\
\text { patients } \\
(n=106)\end{array}$ & Statistics $^{a}$ & $P$-value \\
\hline Age, years & $69.27 \pm 9.47$ & $69.48 \pm 9.15$ & 0.162 & $0.87 \mid$ \\
\hline $\begin{array}{l}\text { Gender (male } \\
\text { proportion) }\end{array}$ & $87.74 \%$ & $87.74 \%$ & 0.000 & 1.000 \\
\hline Smoking percentage & $25.47 \%$ & $88.68 \%$ & 86.429 & $<0.001$ \\
\hline BMI $\left(\mathrm{kg} / \mathrm{cm}^{2}\right)$ & $22.06 \pm 2.95$ & $22.64 \pm 3.32$ & 1.346 & 0.180 \\
\hline $\begin{array}{l}\text { ADAMTS- I3 } \\
(\mathrm{ng} / \mathrm{mL})\end{array}$ & $349.37 \pm 31.88$ & $327.59 \pm 30.7 \mid$ & 5.066 & $<0.001$ \\
\hline vWF (ng/mL) & $4.63 \pm 1.69$ & $9.22 \pm 1.75$ & 19.410 & $<\mathbf{0 . 0 0 1}$ \\
\hline MPV (fl) & $10.26 \pm 0.69$ & $11.82 \pm 0.79$ & $|5.43|$ & $<0.001$ \\
\hline PDW (fl) & $10.19 \pm 1.38$ & $11.01 \pm 1.55$ & 4.020 & $<\mathbf{0 . 0 0 1}$ \\
\hline $\mathrm{Fbg}(\mathrm{g} / \mathrm{L})$ & $2.50 \pm 0.54$ & $4.29 \pm 1.60$ & 10.869 & $<0.001$ \\
\hline D-dimer $(\mu g / L)$ & $292.92(8.83)$ & $295.78(9.61)$ & 2.228 & 0.026 \\
\hline IL-6 (pg/mL) & $2.11(1.17)$ & $3.13(4.12)$ & 3.434 & 0.001 \\
\hline $\mathrm{CRP}(\mathrm{mg} / \mathrm{L})$ & $2.10(1.38)$ & $2.64(4.69)$ & 2.040 & $0.04 I$ \\
\hline $\mathrm{FEV}_{1}$ (\% predicted) & $98.26 \pm 12.15$ & $54.43 \pm 11.27$ & 22.221 & $<\mathbf{0 . 0 0 1}$ \\
\hline $\mathrm{FEV}_{1}(\mathrm{~L})$ & $2.28 \pm 0.47$ & $1.67 \pm 0.74$ & 7.029 & $<0.001$ \\
\hline $\mathrm{FEV}_{1} / \mathrm{FVC}(\%)$ & $76.77 \pm 4.05$ & $57.54 \pm 9.60$ & 19.009 & $<\mathbf{0 . 0 0 1}$ \\
\hline $\begin{array}{l}\text { Proportion of } \\
\text { concealed CRF }\end{array}$ & $7.55 \%$ & $19.81 \%$ & 6.751 & 0.009 \\
\hline
\end{tabular}

Notes: Data are expressed as $n$, mean $\pm S D$, median (interquartile range), or $n(\%)$, unless otherwise stated. aStudent's $t$-test, Wilcoxon rank sum test, or $\chi^{2}$ test was used to find the significant difference between the two groups determined by the type of data. Statistical significance $(P<0.05)$ is shown in bold.

Abbreviations: ADAMTS-13, a disintegrin and metalloproteinase with a thrombospondin type I motif, member 13; BMI, body mass index; CRF, chronic renal failure; CRP, C-reactive protein; Fbg, fibrinogen; $\mathrm{FEV}_{1}$, forced expiratory volume in one second; FVC, forced vital capacity; IL-6, interleukin 6; MPV, mean platelet volume; PDW, platelet distribution width; vWF, von Willebrand factor. under the ROC curves was $0.959(95 \%$ CI $=0.924-0.994$, $P<0.001)$. Sensitivity and specificity at an ADAMTS-13 cutoff value of $318.72 \mathrm{ng} / \mathrm{mL}$ were $100 \%$ and $81.2 \%$, respectively, with a maximum Youden's index.

\section{Discussion}

COPD patients often suffer from several comorbid conditions that may affect the prognosis of COPD at the same time. ${ }^{12}$ However, unlike other comorbidities such as cardiovascular diseases and lung cancer, renal injury in COPD patients is usually neglected, and the number of relevant studies remains limited. A nationwide case-cohort study conducted by Chen and Liao indicated that the overall incidence of CKD was higher in COPD patients (470.9 per 104 person-years).$^{13}$ The incidences of concealed CRF in COPD patients and nonCOPD patients in our study were both lower than those in the study by Incalzi et al reported in 2010, which were $20.8 \%$ and $10.0 \%$, respectively. ${ }^{3}$ Terzano et al had followed 288 Italian COPD patients for 7 years and found that CRF was the second most common comorbidities in COPD (26.3\%), just behind hypertension (64.2\%), but ahead of diabetes $(25.3 \%)$ and cardiac disease $(22.1 \%) .{ }^{14}$ Considering that these studies did not exclude patients who had several conditions that could result in renal injury such as diabetes and included patients who had abnormal serum creatinine level, it was not strange that our study presented a lower incidence of renal failure in COPD patients. The study by Gjerde et al in 2012 had a much lower incidence $(6.9 \%)$ of renal failure in COPD patients from Norway, while the incidence of non-COPD patients was $0.8 \%$, which could be due to many causes, including younger participants and a different eGFR calculation. ${ }^{15}$ Elsawy et al conducted a similar study in 2014, and the incidence of concealed CRF in COPD patients was $17.55 \%$, which was close to our result. ${ }^{16}$ Because of various inclusion criteria and potential regional difference, the incidence of concealed CRF in COPD patients reported remains inconsistent among numerous studies, but it is obvious that COPD patients do have a higher incidence of renal failure though it is often neglected.

Therefore, we conducted this study hoping to explore risk factors for concealed CRF and try to find out an effective biomarker for early diagnosis in COPD patients. It has been reported that COPD patients have an ongoing hypercoagulability state, or put it in other words, prothrombotic state, and a pro-inflammatory state, and a common or similar mechanism exists between inflammation and thrombosis., ${ }^{4,717-20}$ In the same time, both prothrombotic state and inflammation may contribute to the pathogenesis of kidney damage. ${ }^{21}$ Thus, we hypothesized a correlation among prothrombotic state, inflammation, and concealed CRF in COPD patients and 
Table 2 Univariate logistic regression analysis of concealed CRF

\begin{tabular}{|c|c|c|c|c|c|}
\hline & $\begin{array}{l}\text { Normal renal } \\
\text { function }\end{array}$ & $\begin{array}{l}\text { Concealed } \\
\text { CRF }\end{array}$ & OR & $95 \% \mathrm{Cl}$ & $P$-value \\
\hline \multicolumn{6}{|l|}{ All subjects } \\
\hline $\mathrm{n}$ & 183 & 29 & & & \\
\hline Age, years & $69.11 \pm 9.47$ & $71.03 \pm 8.02$ & 1.024 & $0.979-1.070$ & 0.302 \\
\hline Gender (male proportion) & $86.89 \%$ & $93.10 \%$ & 0.491 & $0.110-2.197$ & 0.352 \\
\hline Smoking percentage & $54.64 \%$ & $72.41 \%$ & 2.179 & $0.918-5.173$ & 0.078 \\
\hline BMI $\left(\mathrm{kg} / \mathrm{cm}^{2}\right)$ & $22.31 \pm 3.18$ & $22.62 \pm 2.95$ & 1.031 & $0.912-1.167$ & 0.623 \\
\hline ADAMTS- I3 (ng/mL) & $347.05 \pm 25.70$ & $284.40 \pm 21.01$ & 0.876 & $0.834-0.920$ & $<0.001$ \\
\hline vWF (ng/mL) & $6.63 \pm 2.79$ & $8.77 \pm 2.67$ & 1.331 & $1.135-1.560$ & $<0.001$ \\
\hline MPV (fl) & $10.99 \pm 1.10$ & $11.36 \pm 0.89$ & 1.374 & $0.952-1.983$ & 0.090 \\
\hline PDW (fl) & $10.60 \pm 1.52$ & $10.61 \pm 1.54$ & 1.007 & $0.778-1.302$ & 0.960 \\
\hline Fbg $(g / L)$ & $3.16 \pm 1.10$ & $4.91 \pm 2.49$ & 1.935 & $|.49|-2.5 \mid 2$ & $<0.001$ \\
\hline D-dimer $(\mu \mathrm{g} / \mathrm{L})$ & $293.70(7.80)$ & $317.28(13.12)$ & 1.146 & $1.097-1.198$ & $<0.001$ \\
\hline IL-6 (pg/mL) & $2.22(1.86)$ & $3.04(3.95)$ & 1.100 & $0.977-1.239$ & 0.116 \\
\hline CRP (mg/L) & $2.07(1.77)$ & $5.05(8.02)$ & 1.218 & I.102-1.345 & $<0.001$ \\
\hline $\mathrm{FEV}_{1}$ (\% predicted) & $77.73 \pm 26.05$ & $67.59 \pm 26.06$ & 0.985 & $0.97 I-1.000$ & 0.055 \\
\hline $\mathrm{FEV}_{1}(\mathrm{~L})$ & $2.00 \pm 0.68$ & $1.82 \pm 0.75$ & 0.689 & $0.391-1.213$ & 0.197 \\
\hline $\mathrm{FEV}_{1} / \mathrm{FVC}(\%)$ & $67.07 \pm 12.26$ & $67.72 \pm 11.40$ & 1.005 & $0.972-1.038$ & 0.785 \\
\hline Proportion of COPD & $46.45 \%$ & $72.41 \%$ & 3.026 & $1.275-7.185$ & 0.012 \\
\hline \multicolumn{6}{|l|}{ COPD subjects } \\
\hline $\mathrm{n}$ & 85 & 21 & & & \\
\hline Age, years & $69.13 \pm 9.51$ & $70.90 \pm 7.56$ & 1.023 & $0.967-1.082$ & 0.426 \\
\hline Gender (male proportion) & $87.06 \%$ & $90.48 \%$ & 0.708 & $0.145-3.468$ & 0.670 \\
\hline Smoking percentage & $88.24 \%$ & $90.48 \%$ & 1.267 & $0.256-6.270$ & 0.772 \\
\hline BMI $\left(\mathrm{kg} / \mathrm{cm}^{2}\right)$ & $22.67 \pm 3.35$ & $22.52 \pm 3.25$ & 0.986 & $0.853-1.140$ & 0.850 \\
\hline ADAMTS-I3 (ng/mL) & $337.91 \pm 23.22$ & $285.85 \pm 20.15$ & 0.891 & $0.846-0.937$ & $<0.001$ \\
\hline vWF (ng/mL) & $8.99 \pm 1.80$ & $10.14 \pm 1.14$ & 1.525 & $1.105-2.104$ & 0.010 \\
\hline MPV (fl) & $11.86 \pm 0.80$ & $1 \mathrm{l} .67 \pm 0.73$ & 0.730 & $0.390-1.367$ & 0.325 \\
\hline PDW (fl) & $11.02 \pm 1.58$ & $10.97 \pm 1.48$ & 0.980 & $0.719-1.337$ & 0.900 \\
\hline Fbg $(g / L)$ & $3.93 \pm 1.11$ & $5.74 \pm 2.35$ & 2.152 & I.47I-3.I49 & $<0.001$ \\
\hline D-dimer $(\mu \mathrm{g} / \mathrm{L})$ & $294.38(7.49)$ & $317.28(13.12)$ & 1.126 & $1.07 \mid-1.184$ & $<0.001$ \\
\hline IL-6 (pg/mL) & $3.10(4.15)$ & $3.19(4.66)$ & 1.006 & $0.879-1.15 \mid$ & 0.934 \\
\hline CRP (mg/L) & $2.22(2.65)$ & $8.22(9.17)$ & 1.166 & $1.055-1.288$ & 0.003 \\
\hline $\mathrm{FEV}_{1}$ (\% predicted) & $54.14 \pm 15.97$ & $55.62 \pm 17.79$ & 1.006 & $0.976-1.036$ & 0.708 \\
\hline $\mathrm{FEV}_{1}(\mathrm{~L})$ & $1.67 \pm 0.73$ & $|.7| \pm 0.80$ & 1.080 & $0.567-2.057$ & 0.815 \\
\hline $\mathrm{FEV}_{\mathrm{I}} / \mathrm{FVC}(\%)$ & $57.78 \pm 9.33$ & $56.57 \pm 10.83$ & 0.987 & $0.941-1.036$ & 1.036 \\
\hline
\end{tabular}

Notes: Data are expressed as $n$; mean \pm SD; median (interquartile range); or $n(\%)$, unless otherwise stated. Statistical significance $(P<0.05)$ is shown in bold.

Abbreviations: ADAMTS-13, a disintegrin and metalloproteinase with a thrombospondin type I motif, member I3; BMI, body mass index; CRF, chronic renal failure; CRP, C-reactive protein; Fbg, fibrinogen; FEV , forced expiratory volume in one second; FVC, forced vital capacity; IL-6, interleukin 6; MPV, mean platelet volume; OR, odds ratio; PDW, platelet distribution width; vWF, von Willebrand factor.

Table 3 Multivariable logistic regression analysis of concealed CRF

\begin{tabular}{lcll}
\hline & OR & $\mathbf{9 5 \%} \mathbf{C l}$ & P-value \\
\hline All subjects & & & \\
ADAMTS-13 & 0.858 & $0.795-0.926$ & $<\mathbf{0 . 0 0 1}$ \\
D-dimer & 1.095 & $1.027-1.169$ & $\mathbf{0 . 0 0 6}$ \\
CRP & 1.252 & $1.058-1.480$ & $\mathbf{0 . 0 0 9}$ \\
COPD subjects & & & \\
ADAMTS-13 & 0.814 & $0.702-0.944$ & $\mathbf{0 . 0 0 7}$ \\
D-dimer & 1.141 & $1.028-1.266$ & $\mathbf{0 . 0 1 3}$ \\
CRP & 1.361 & $1.050-1.764$ & $\mathbf{0 . 0 2 0}$ \\
\hline
\end{tabular}

Note: Statistical significance $(P<0.05)$ is shown in bold.

Abbreviations: ADAMTS-13, a disintegrin and metalloproteinase with a thrombospondin type I motif, member 13; CRF, chronic renal failure; CRP, C-reactive protein; OR, odds ratio. investigated it by analyzing several clinical indicators associated with prothrombotic state and inflammation. MPV is thought to be associated with thrombosis and inflammation. An increase in MPV is a risk factor for thrombosis, and a mild inflammation status is usually accompanied with an increase in MPV, while severe inflammation is opposite..$^{22}$ Some studies show that deep vein thrombosis patients who had high levels of MPV and PDW are susceptible to pulmonary embolism. ${ }^{23}$ The relation between thrombosis and increase in Fbg and D-dimer is well known. ${ }^{24,25}$ AbdelHalim and AboElNaga 


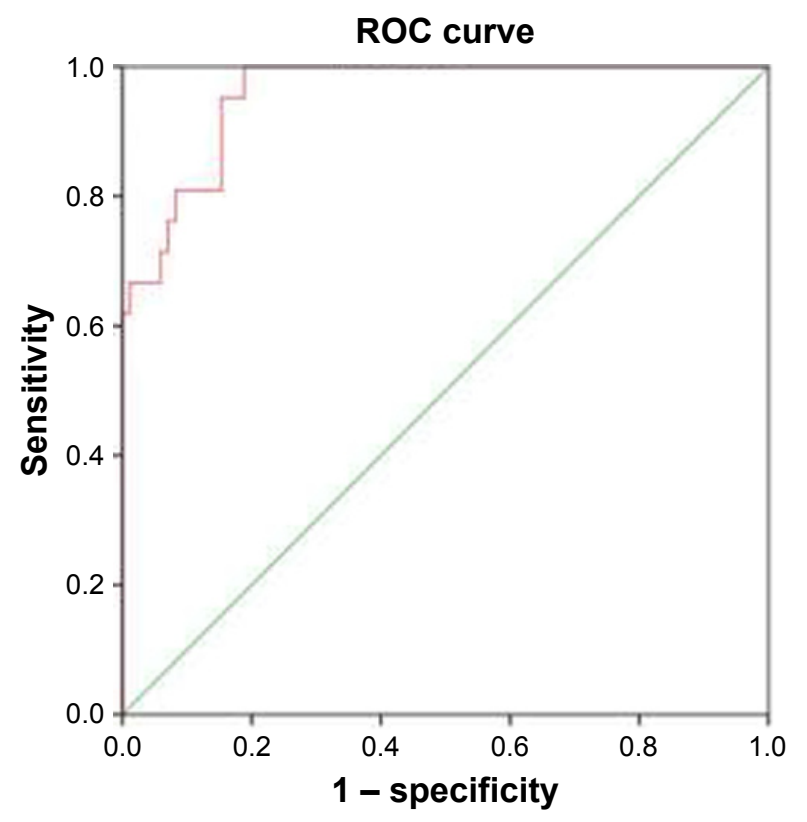

Figure I ROC curves for ADAMTS-I3 in predicting concealed CRF in COPD patients. The area under the ROC curve was 0.959 . Sensitivity and specificity at an ADAMTS- 13 cutoff of $318.72 \mathrm{ng} / \mathrm{mL}$ were $100 \%$ and $81.2 \%$, respectively.

Abbreviations: ADAMTS-13, a disintegrin and metalloproteinase with a thrombospondin type I motif, member 13; CRF, chronic renal failure; ROC, receiver operating characteristic.

reported that COPD patients had significantly higher D-dimer levels, even those with low-probability revised Geneva scores and negative computed tomography pulmonary angiogram findings for pulmonary embolism. ${ }^{26}$ In addition, vWF, a key initiator of the clotting cascade, is closely relevant to hypercoagulability and thrombosis. ${ }^{27}$ Wedzicha et al found that the acute exacerbation of COPD could cause the increase of IL-6 and then stimulate the increase of Fbg. ${ }^{28}$ The increase of CRP is also a risk factor for thrombosis and serves as an inflammatory marker. ${ }^{29,30}$ Therefore, clinical indicators mentioned above were analyzed in our study. In addition, ADAMTS-13 is closely related to thrombosis and inflammation by splitting vWF and downregulating thrombosis and neutrophilic infiltrates. ${ }^{9,31}$ A case report indicated that ADAMTS-13 deficiency should be considered a cause of CKD. ${ }^{32}$ Therefore, we conjectured that ADAMTS-13 may be a potential predictor of concealed CRF in COPD patients. As shown in Table 1, COPD patients are accompanied with higher levels of $v W F$, MPV, PDW, Fbg, D-dimer, IL-6, and CRP, but a lower level of ADAMTS-13, indicating that these prothrombotic state and inflammation relevant factors might contribute to a higher incidence of concealed CRF in COPD patients; as expected, when grouped according to renal function, the patients with concealed CRF had higher levels of vWF, Fbg, D-dimer, and CRP, but a lower level of ADAMTS-13 (Table 2).
After correction of confounding variables, it presented D-dimer ( $\mathrm{OR}=1.095,95 \% \mathrm{CI}=1.027-1.169)$ and $\mathrm{CRP}$ (OR $=1.252,95 \% \mathrm{CI}=1.058-1.480)$ as risk factors while ADAMTS- $13(\mathrm{OR}=0.858,95 \% \mathrm{CI}=0.795-0.926)$ as a protective factor of concealed CRF, which was consistent with our expectations. The subgroup analysis of COPD subjects showed a similar result, while both the two analyses did not reveal an association between spirometry data and concealed CRF reported by AbdelHalim and AboElNaga. ${ }^{33}$ We further investigated the predictive value of ADAMTS-13 on predicting concealed CRF in COPD patients using ROC curves, and the results showed that it possessed rather a high diagnostic value (Figure 1).

Most relevant studies mentioned above explored risk factors for CRF by focusing on age, sex, smoking history, nutritional status, and pulmonary function. However, as far as it is concerned, there is no report studying the relation among prothrombotic state, inflammation, and $\mathrm{CRF}$ in $\mathrm{COPD}$ patients. Thus, apart from factors mentioned in other studies, for the first time, we focused on the possible link among prothrombotic state, inflammation, and CRF and explored the predictive values of ADAMTS-13 on concealed CRF in COPD patients, hoping to seek for an effective biomarker for early diagnosis. Although the present study has yielded some preliminary findings, we can nevertheless confirm that there are some limitations in this study. First, as an observational study, it included small samples obtained from a single geographic facility. Second, we selected several clinical indicators only relevant to prothrombotic state and inflammation, but many other coagulation indicators and inflammation indicators were not included.

\section{Conclusion}

Concealed CRF is prevalent in COPD patients, which might be attributed to prothrombotic state and systematic status, and the determination of ADAMTS-13 may benefit early diagnosis of concealed CRF in COPD patients. However, prospective trials with better design and larger sample sizes are needed to overcome the limitations of our study.

\section{Availability of data and materials}

The data sets used and/or analyzed during the current study are available from the corresponding author on a reasonable request.

\section{Acknowledgments}

This study was supported by the Science and Technology Planning Project of Guangzhou (2014Y2-00136); the Science 
and Technology Planning Project of Guangdong Province, China (2014A020212151); and the Major Science and Technology Planning Project of Guangdong Province, China (2016A020216009).

\section{Author contributions}

All authors contributed toward data analysis, drafting and critically revising the paper, gave final approval of the version to be published, and agree to be accountable for all aspects of the work.

\section{Disclosure}

The authors report no conflicts of interest in this work.

\section{References}

1. Global initiative for chronic obstructive lung disease, Inc. Global strategy for the diagnosis, management and prevention of chronic obstructive pulmonary disease. 2011 report; 2011 [updated 2014]. Available from: http://goldcopd.org. Accessed May 23, 2014.

2. Gaddam S, Gunukula SK, Lohr JW, Arora P. Prevalence of chronic kidney disease in patients with chronic obstructive pulmonary disease: a systematic review and meta-analysis. BMC Pulm Med. 2016; 16(1):158.

3. Incalzi RA, Corsonello A, Pedone $\mathrm{C}$, et al. Chronic renal failure: a neglected comorbidity of COPD. Chest. 2010;137(4):831-837.

4. Alessandri C, Basili S, Violi F, Ferroni P, Gazzaniga PP, Cordova C. Hypercoagulability state in patients with chronic obstructive pulmonary disease. Chronic Obstructive Bronchitis and Haemostasis Group. Thromb Haemost. 1994;72(3):343-346.

5. Ashitani J, Mukae H, Arimura Y, Matsukura S. Elevated plasma procoagulant and fibrinolytic markers in patients with chronic obstructive pulmonary disease. Intern Med. 2002;41(3):181-185.

6. Eagan TM, Ueland T, Wagner PD, et al. Systemic inflammatory markers in COPD: results from the Bergen COPD Cohort Study. Eur Respir J. 2010;35(3):540-548.

7. Gan WQ, Man SF, Senthilselvan A, Sin DD. Association between chronic obstructive pulmonary disease and systemic inflammation: a systematic review and a meta-analysis. Thorax. 2004;59(7):574-580.

8. Tsai HM. ADAMTS13 and microvascular thrombosis. Expert Rev Cardiovasc Ther. 2006;4(6):813-825.

9. Chauhan AK, Kisucka J, Brill A, Walsh MT, Scheiflinger F, Wagner DD. ADAMTS13: a new link between thrombosis and inflammation. $J$ Exp Med. 2008;205(9):2065-2074.

10. Samama MM, Horellou MH, Elalamy I, Mathieux V, OmbandzaMoussa E, Conard J. D-dimer levels, constitutional thrombophilia, and venous thrombosis prediction: clinical aspects and implications. Semin Vasc Med. 2005;5(4):371-374.

11. Levey AS, Stevens LA, Schmid CH, et al. A new equation to estimate glomerular filtration rate. Ann Intern Med. 2009;150(9):604-612.

12. Cavailles A, Brinchault-Rabin G, Dixmier A, et al. Comorbidities of COPD. Eur Respir Rev. 2013;22(130):454-475.

13. Chen CY, Liao KM. Chronic obstructive pulmonary disease is associated with risk of chronic kidney disease: a nationwide case-cohort study. Sci Rep. 2016;6:25855.
14. Terzano C, Conti V, Di Stefano F, et al. Comorbidity, hospitalization, and mortality in COPD: results from a longitudinal study. Lung. 2010;188(4):321-329.

15. Gjerde B, Bakke PS, Ueland T, Hardie JA, Eagan TM. The prevalence of undiagnosed renal failure in a cohort of COPD patients in western Norway. Respir Med. 2012;106(3):361-366.

16. Elsawy S, Abu Bakr S, Oraby S, Saied T, Abu Elkhair O. Prediction of chronic renal failure in patients with chronic obstructive pulmonary disease. Eur Respir J. 2014;44(Suppl 58):P3627.

17. Abd Elsalam HM, Mohamed MA, El Gammal MS, El-Shabrawy M. Hypercoagulability in different respiratory diseases. Egypt J Chest Dis Tuberc. 2013;62(2):331-341.

18. Song YJ, Zhou ZH, Liu YK, Rao SM, Huang YJ. Prothrombotic state in senile patients with acute exacerbations of chronic obstructive pulmonary disease combined with respiratory failure. Exp Ther Med. 2013; 5(4):1184-1188.

19. King PT. Inflammation in chronic obstructive pulmonary disease and its role in cardiovascular disease and lung cancer. Clin Transl Med. 2015;4(1):68.

20. Esmon CT. Crosstalk between inflammation and thrombosis. Maturitas. 2008;61(1-2):122-131.

21. Kazancioglu R. Risk factors for chronic kidney disease: an update. Kidney Int Suppl (2011). 2013;3(4):368-371.

22. Gasparyan AY, Ayvazyan L, Mikhailidis DP, Kitas GD. Mean platelet volume: a link between thrombosis and inflammation? Curr Pharm Des. 2011;17(1):47-58.

23. Sevuk U, Bahadir MV, Altindag R, et al. Value of serial platelet indices measurements for the prediction of pulmonary embolism in patients with deep venous thrombosis. Ther Clin Risk Manag. 2015;11: 1243-1249.

24. de Moerloose P, Boehlen F, Neerman-Arbez M. Fibrinogen and the risk of thrombosis. Semin Thromb Hemost. 2010;36(1):7-17.

25. Stein PD, Hull RD, Patel KC, et al. D-dimer for the exclusion of acute venous thrombosis and pulmonary embolism: a systematic review. Ann Intern Med. 2004;140(8):589-602.

26. AbdelHalim HA, AboEINaga HH. Acute exacerbation of COPD with pulmonary embolism: a new D-dimer cut-off value. Egyp J Chest Dis Tuberc. 2017;66(2):227-230.

27. Dmitrieva NI, Burg MB. Secretion of von Willebrand factor by endothelial cells links sodium to hypercoagulability and thrombosis. Proc Natl Acad Sci U S A. 2014;111(17):6485-6490.

28. Wedzicha JA, Seemungal TA, MacCallum PK, et al. Acute exacerbations of chronic obstructive pulmonary disease are accompanied by elevations of plasma fibrinogen and serum IL-6 levels. Thromb Haemost. 2000;84(2):210-215.

29. Fay WP. Linking inflammation and thrombosis: role of C-reactive protein. World J Cardiol. 2010;2(11):365-369.

30. Agarwal R, Zaheer MS, Ahmad Z, Akhtar J. The relationship between $\mathrm{C}$-reactive protein and prognostic factors in chronic obstructive pulmonary disease. Multidiscip Respir Med. 2013;8(1):63.

31. Feng Y, Li X, Xiao J, et al. ADAMTS13: more than a regulator of thrombosis. Int J Hematol. 2016;104(5):534-539.

32. Bramham K, Hilton R, Horsfield C, McDonald V, Camilleri R, Hunt BJ. ADAMTS-13 deficiency: can it cause chronic renal failure? Nephrol Dial Transplant. 2011;26(2):742-744.

33. AbdelHalim HA, AboElNaga HH. Is renal impairment an anticipated COPD comorbidity? Respir Care. 2016;61(9):1201-1206. 
International Journal of COPD

\section{Publish your work in this journal}

The International Journal of COPD is an international, peer-reviewed journal of therapeutics and pharmacology focusing on concise rapid reporting of clinical studies and reviews in COPD. Special focus is given to the pathophysiological processes underlying the disease, intervention programs, patient focused education, and self management protocols.

This journal is indexed on PubMed Central, MedLine and CAS. The manuscript management system is completely online and includes a very quick and fair peer-review system, which is all easy to use. Visit http://www.dovepress.com/testimonials.php to read real quotes from published authors 